\title{
Spectral behavior of phase scintillation in the nighttime auroral region
}

\author{
E. J. Fremouw, J. A. Secan, and J. M. Lansinger \\ Physical Dynamics, Inc., Bellevue, Washington \\ (Received December 10, 1984; revised February 18, 1985; accepted February 18, 1985.)
}

\begin{abstract}
Enhancement of phase and intensity scintillation, as a radio line of sight scans through grazing incidence on the local $L$ shell in the nightside diffuse-auroral ionosphere, has been well documented by means of data from the DNA Wideband satellite. In this paper we describe systematic behavior of the phase spectrum found in the enhancement region over Poker Flat, Alaska. Routine Wideband processing included spectral analysis of $20-\mathrm{s}(\sim 60-\mathrm{km})$ segments of VHF and UHF phase records and log linear fits thereto. Tabulation of the resulting power law spectral indices, $p$, disclosed increased values in the scintillation enhancement region. It has been established that the increase in $p$ is the signature of a physically real phenomenon and not merely an artifact of statistical nonstationarity arising from the narrowness of the scintillation strength enhancement. Moreover, it has been found that $p$ is not increased in strength enhancements occurring close to the magnetic zenith. Indeed, in some cases, it is substantially decreased. A possible source of these unexpected spectral behaviors is size-dependent anisotropy, an idealization of the irregularities responsible being small-scale field-aligned rods imbedded in large-scale shell-aligned sheets. We also present examples of multiple-regime power law spectra, characterized by an increased spectral index at short structure wavelengths (less than a few hundred meters north-south) and, more frequently, by an increased index at large wavelengths (more than a few kilometers). These spectral breaks occur both separately and together and both within and outside the scintillation enhancement region.
\end{abstract}

\section{INTRODUCTION}

There is interest in the shape of phase scintillation spectra for some systems applications and, geophysically, because of its relationship to the spectrum of refractive-index irregularities responsible for scintillation. The relationship is most directly seen between the Fourier transforms of the spectra. For ionospheric studies, the general relationship of interest is between the two-dimensional autocorrelation function, $R_{\phi}(\zeta)$, of phase on an observing plane and the three-dimensional autocorrelation function, $R_{\Delta N}(\zeta, \eta)$, of plasma density irregularities [e.g., Rino and Fremouw, 1977], weighted along the line of sight as follows:

$$
R_{\phi}(\zeta ; L)=r_{e}^{2} \lambda^{2} L \int_{-L}^{L}\left(1-\frac{|\eta|}{L}\right) R_{\Delta N}(\zeta, \eta) d \eta
$$

where $r_{e}$ is the classical electron radius, $\lambda$ is the radio wavelength, and $L$ is the half thickness of the irregular medium.

For a medium that is many correlation distances thick, the weighting factor reduces to unity, and the

Copyright 1985 by the American Geophysical Union.

Paper number 5 S0146.

$0048-6604 / 85 / 005 S-0146 \$ 08.00$ integration limits go effectively to infinity. Then (but only in this case of a thick medium), the spatial integration averages out all but the dc term of the lineof-sight wave number spectrum. For power law spectra, the result is well known: the two-dimensional index of the ac phase spectrum is the same as the three-dimensional index of the irregularity spectrum.

A strip scan on the observing plane (as, for instance, when the phase pattern from a low-orbiting satellite sweeps across a receiving antenna) is made at zero lag in the dimension perpendicular to the scan, which is tantamount to a one-dimensional integration of the two-dimensional spectrum. The power law index then is reduced by unity to, say, $p$. In contrast, a one-dimensional scan of the irregularities in situ effectively integrates the three-dimensional spectrum over two dimensions [Cronyn, 1970], reducing the index by 2 to, say, $q=p-1$.

Scintillation researchers exploit the above simple relationship between the phase spectral index, $p$, and the in situ index, $q$, to identify the spectral shape of plasma density irregularities. Direct comparisons also have been made between phase scintillation and in situ spectral measurements [e.g., Rino et al., 1981], mutual consistency being found. The measurements also have disclosed departures from simple power 


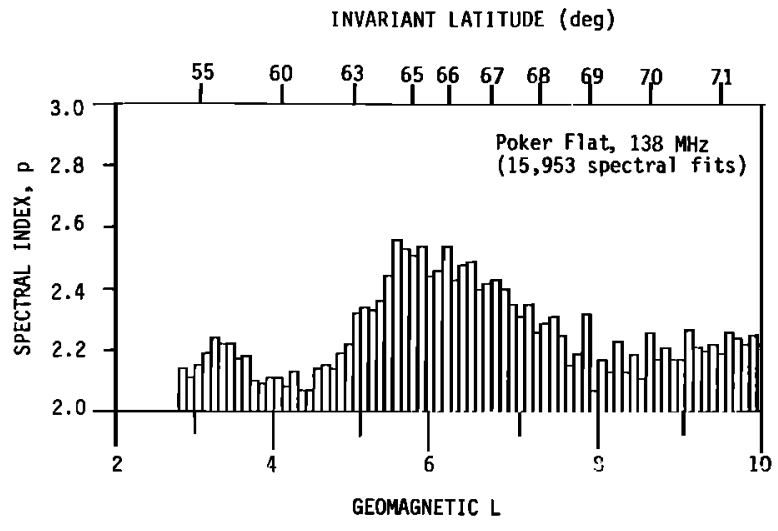

Fig. 1. Relationship between VHF phase spectral index, $p$, and geomagnetic latitude (and $L$ value) at the line-of-sight penetration point in the $F$ layer.

law behavior. Rino et al. [1981] and Basu et al. [1983] have found breaks in the power law spectrum of equatorial irregularities at structure wavelengths of the order of $1 \mathrm{~km}$, with steeper (shallower) spectral indices for smaller (larger) structures.

Recently, Basu et al. [1984] have reported similar breaks in in situ spectra from two orbits of AE-D, at somewhat smaller wavelengths, in regions of large auroral acceleration events. They suggest that such breaks may result from damping of $F$ region irregularities by high $E$ layer conductivity [Vickrey and Kelley, 1982]. In the present work, we have found similar breaks in about a quarter of approximately 200 nighttime phase spectra from Poker Flat, Alaska, obtained by means of the DNA Wideband satellite, which we had carefully processed and inspected for such features. More frequently (in about three quarters of the spectra), we found "inverse" breaks at larger scan direction wavelengths (a few to several kilometers), with steeper (shallower) indices for larger (smaller) structures. The two breaks also occur together, resulting in a three-component (steep, shallow, steep) spectrum between scan direction wavelengths of $30 \mathrm{~km}$ (limited by detrending) and about $100 \mathrm{~m}$ (limited by the noise floor).

In this paper, we give more attention to a systematic behavior of the midrange spectral index of phase scintillation (approximately between $6 \mathrm{~km}$ and $300 \mathrm{~m}$ wavelength). We find that it steepens in the region of the subauroral scintillation enhancement [Fremouw et al., 1977] for nonoverhead passes but not for passes close to the receiver's magnetic meridian. We interpret this as a geometrical effect stemming from a scale-size dependence of cross-field anisotropy of the irregularities. We also point out a strictly geophysical (as contrasted with geometrical) alternative explanation and recognize the possibility of contamination by violation of the usual thick-medium limit of equation (1).

\section{DATA BASE AND ITS PROCESSING}

Our data base comprises spectra of VHF phase scintillations recorded (using $S$ band as a phase reference) during Wideband passes over Poker Flat ( $F$ region invariant latitude $=65.7^{\circ}$ ) between May of 1976 and February of 1979 . Routine processing of Wideband data was described by Fremouw et al. [1978]. It included spectral analysis, without tapered windowing in the time domain or spectral smoothing in the frequency domain, of selected 20-s intensity and phase records after removal of trends with Fourier periods greater than $10 \mathrm{~s}$ (corresponding to about $30 \mathrm{~km}$ of scan through the $F$ layer at $350 \mathrm{~km}$ ). The phase spectra were routinely characterized by a single power law spectral index, $p$, obtained by a log linear best fit between fluctuation frequencies of 0.5 and $10 \mathrm{~Hz}$ (scan wavelengths of about $6 \mathrm{~km}$ and 300 $\mathrm{m}$, respectively).

Routine spectral analysis was performed in Wideband essentially whenever measurable scintillation was observed, the weakest scintillation events being most susceptible to noise-floor contamination (suppression of $p$ values). Leaving out passes for which the local (College) magnetic $K$ index was $<1$, which tended to correspond to the weakest scintillation, we have ordered all other best fit $p$ values for nighttime passes from May of 1976 through January of 1979 against the magnetic latitude of the line-of-sight penetration point with the $F$ layer $(350 \mathrm{~km})$ in Figure 1. Containing spectral indices from 15,953 routine best fits, the figure shows a systematic increase in $p$ (steepening of the spectrum) near the region of Poker Flat's local $L$ shell scintillation enhancement. Including all nighttime data $(17,937$ spectra from May of 1976 through January of 1979 , with no $K$ index threshold) produced little change in the latitudinal behavior of $p$.

We were surprised by the pattern illustrated in Figure 1 and were concerned that it might be an artifact of statistical nonstationarity stemming from the localized increase in scintillation strength in the enhancement region. To test this possibility, we generated simulated data by adding a large number of sine waves weighted according to a prescribed power law and having randomly related phases. We simulated a local scintillation enhancement by multi- 


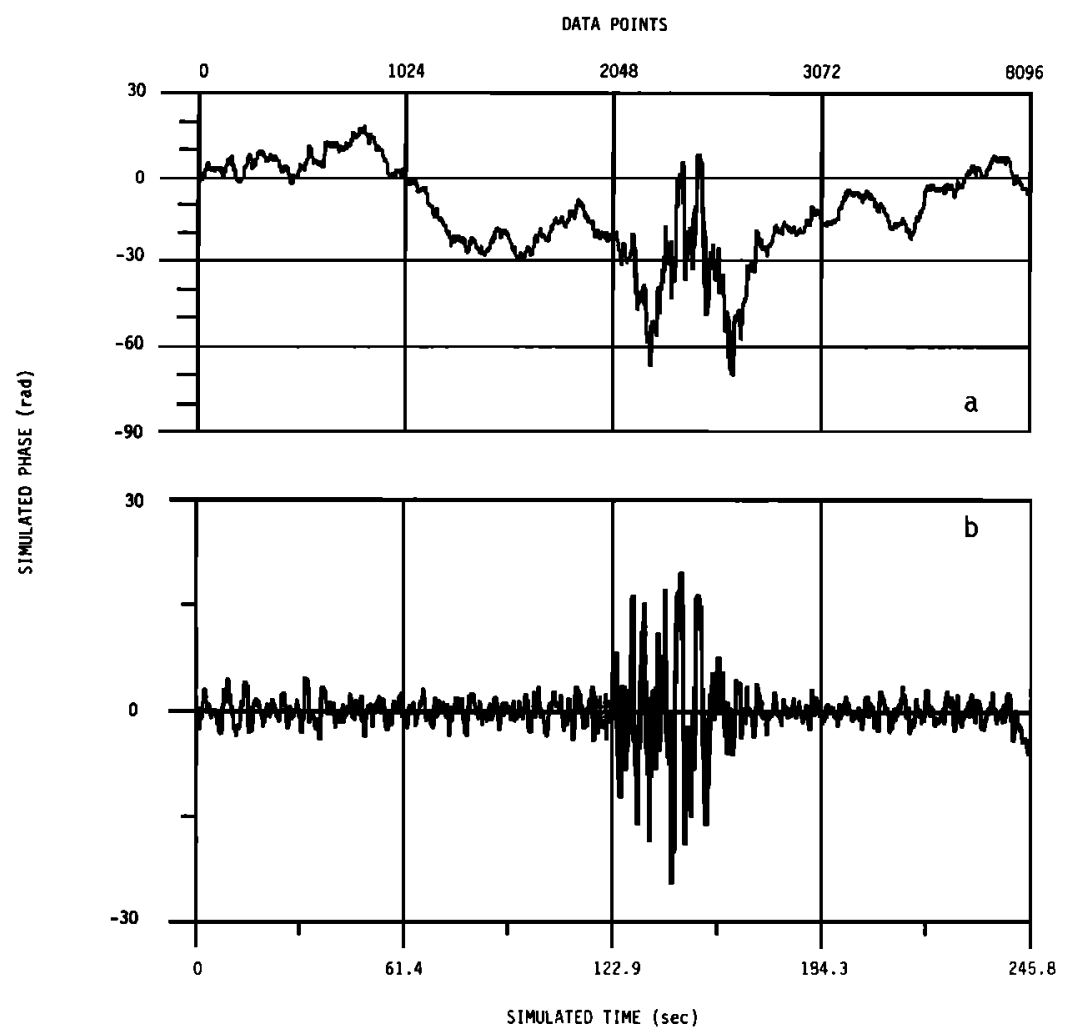

F1g. 2. Simulated phase scintillation data. (a) Time series generated from two-component power law spectrum, enhanced by a multiplicative gaussian centered at time point 2426. (b) Same time series after detrending in manner employed in Wideband experiment.

plying the resulting 8096-point time series by unity plus a fivefold gaussian, centered at 2426 points and having a $1 / e$ width of 512 points. The resulting locally enhanced time series appears in Figure $2 a$. We then detrended the time series in a manner scaled to emulate routine Wideband processing, the detrended result appearing in Figure $2 b$.

Adjacent sections of the detrended waveform, outside of and through the simulated scintillation enhancement, then were spectrally analyzed. Several other time series also were produced, by means of different detrend cutoffs, and spectrally analyzed with windows twice as long as the cutoff period, as had been done routinely in Wideband. The point was to obtain processing windows shorter than, longer than, and comparable to the enhancement duration. In no case did the nonstationarity represented by the enhancement distort the recovered spectrum, as is illustrated in Figure 3.

The lower spectrum in Figure 3 is from a 256point section of the time series in Figure $2 b$, from well outside the simulated enhancement. The super- imposed straight lines designate the prescribed spectrum, which contained a break to simulate those found near the equator by Rino et al. [1981] and Basu et al. [1983] and recently found in auroral acceleration regions by Basu et al. [1984]. The upper spectrum is from the 256-point section of the time series centered on the enhancement, and the straight lines on it designate the specified power spectrum times 36 , the square of the gaussian enhancement factor.

Note that the processed spectrum fits the input spectrum just as well in the enhancement as outside of it. The nonstationarity of the data has introduced no spectral distortion. Again, this was true for window sizes comparable to and larger than the gaussian width as well as for the smaller window used for Figure 3, and it was as true in transition sections as in the fully enhanced and totally nonenhanced sections.

The one significant manner in which the spectral processing leading to Figure 3 differs from that used routinely in Wideband is that the time series shown 


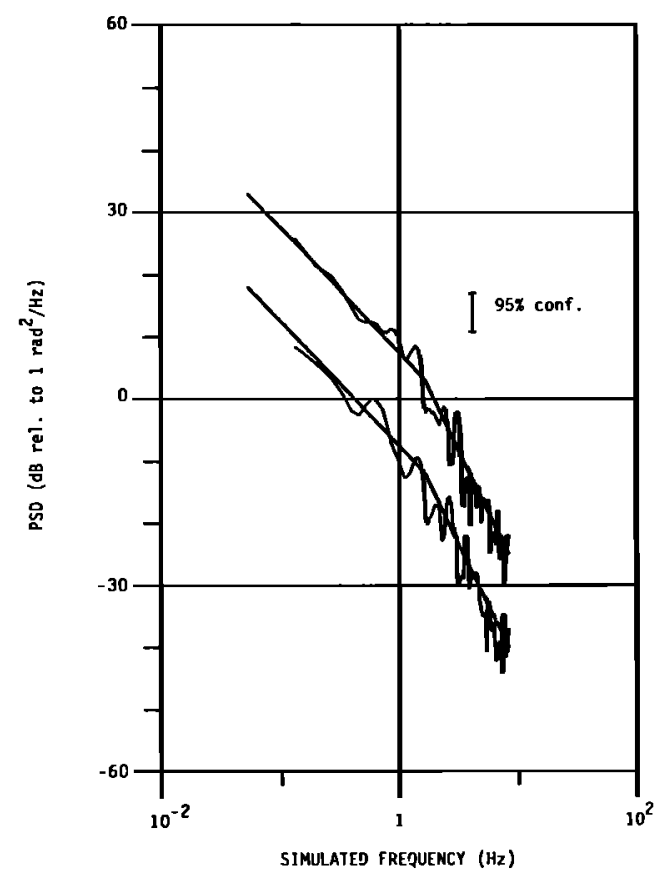

Fig. 3. Spectra of two 256-point segments of Figure $2 b$; upper centered on simulated enhancement, lower well away from it. Solid lines indicate enhanced and nonenhanced spectrum from which Figure 2 was generated. Note complete lack of any spectral distortion introduced by nonstationarity of enhancement.

in Figure $2 b$ was passed through a tapered window prior to being discrete-Fourier transformed. In tests with simulated data and with routinely and specially processed Wideband data, we found that lack of a tapered window (i.e., use of a de facto rectangular window) frequently produces a troublesome level of spectral leakage in phase scintillation spectra. Specifcally, we found that spectral features such as the breaks shown in the simulated data of Figure 3 could be totally obscured by leakage stemming from phase discontinuities at the ends of data windows even after detrending. It is this, we believe, that prevented identification of spectral breaks earlier in the Wideband program and possibly in other scintillation data sets. We found no evidence, however, that any processing artifact caused the systematic variation of the midrange spectral index, $p$, shown in Figure 1. All data specially processed for this study were passed through a 74-dB Blackman-Harris window [Harris, 1978] prior to spectral analysis.

\section{SPECTRAL BEHAVIOR}

Features observed. Having established that routine Wideband processing of even nonstationary data does not steepen the midrange spectrum (leakage being expected to flatten it, if anything), we proceeded to investigate the systematic increase in $p$ noted in Figure 1. Figure 4 illustrates this behavior in a typical case. The lower spectrum is from $20 \mathrm{~s}$ just prior to (poleward of) an observed scintillation enhancement, and the upper one is from the next $20 \mathrm{~s}$, just after abrupt onset of the enhancement. Here, the straight lines are best fits between 0.5 and $10 \mathrm{~Hz}$ (approximately $6 \mathrm{~km}$ and $300 \mathrm{~m}$, respectively, in the essentially cross- $L$ shell scan direction). They correspond to $p$ values of 2.2 and 2.9 outside and inside the enhancement, respectively. That is, the enhancement favors larger-scale structures.

There is another feature in Figure 4 that was not present in the simulation data illustrated in Figure 3. Both the enhanced and nonenhanced spectra break away upward from the middle-frequency best fit at the lowest frequencies. We noted this behavior in $77 \%$ of the 214 nighttime spectra that we've carefully inspected. It is not confined to the region of geometrical enhancement. We also carefully checked, by means of simulated data, the possibility that it might

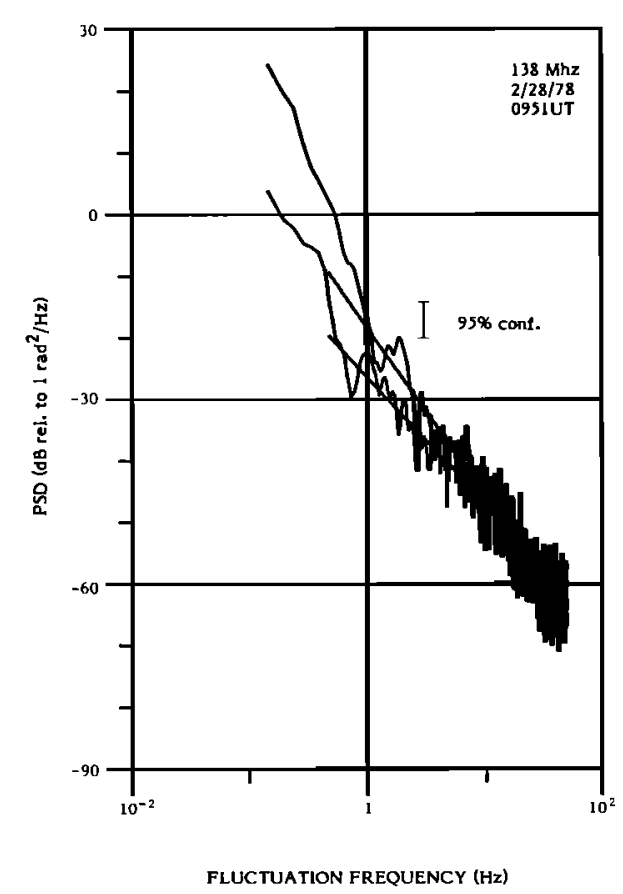

Fig. 4. Typical spectra from just inside (upper) and just outside (lower) the subauroral scintillation enhancement. Note (1) the increase in midrange spectral index, $p$, from 2.2 outside to 2.9 inside the enhancement and (2) the upward break of both spectra away from the midrange best fit at the lowest frequencies (structures larger than a few kilometers in approximately the magnetic northsouth scan direction). 
be the convolution signature of our processing window; it is not.

Now, Figure 4 does not contain the downward break at high frequencies identified in in situ auroralionosphere data by Basu et al. [1984]. Our scintillation data base does contain examples of such behavior, however, and it is illustrated in Figure 5, along with the "inverse" (upward) break shown in Figure 4. The high-frequency downward break was found in $23 \%$ of our 214 specially processed and carefully inspected spectra. As does its low-frequency inverse counterpart, it occurs both inside and outside the scintillation enhancement region. Thus, both types and senses of spectral break are of geophysical origin and may be expected in in situ data. We suggest, on the other hand, that the midrange steepening observed only in scintillation enhancements is geometrically imposed, albeit a conveyer of geophysical information, and will not be found in in situ data.

Systematic trends. Our reason for supposing the midrange steepening to be of geometrical origin arises upon closer examination of the behavior shown in Figure 1. To make such an examination, we investigated the two-dimensional character of the observed systematic increase in $p$. Specifically, for each $p$ measurement, we computed the angle between the radio line of sight and (1) the geomagnetic $L$ shell and (2) the magnetic meridian, both at a height of $350 \mathrm{~km}$. We then made a contour plot of average $p$ values on a grid of off-shell and off-meridian angle.

To test the statistical significance of the $L$ shellaligned band of increased $p$ that we expected to find at night, we performed the same analysis of daytime data, for which we did not expect such a pattern. Figure 6 contains a contour plot of $p$ values from all 6647 daytime spectra available within the look-angle window employed. The grid is oriented as if one were looking down on a map of Alaska. That is, positive off-meridian angles correspond to satellite locations to the (geomagnetic) east of Poker Flat, and positive off-shell angles correspond to northerly satellite positions. As expected, there is no discernible band aligned with the local $L$ shell (off-shell angle $=0$ ). Indeed, there is no systematic pattern whatever.

The absence of a region of increased $p$ near the magnetic zenith $(0,0$ on the plot) represents independent evidence that the increase observed at night is not an artifact of nonstationarity. Plasma density irregularities, including those over Poker Flat in the daytime [Fremouw and Lansinger, 1981], are elongated along the magnetic field, producing a geometrical enhancement of scintillation strength for lines of

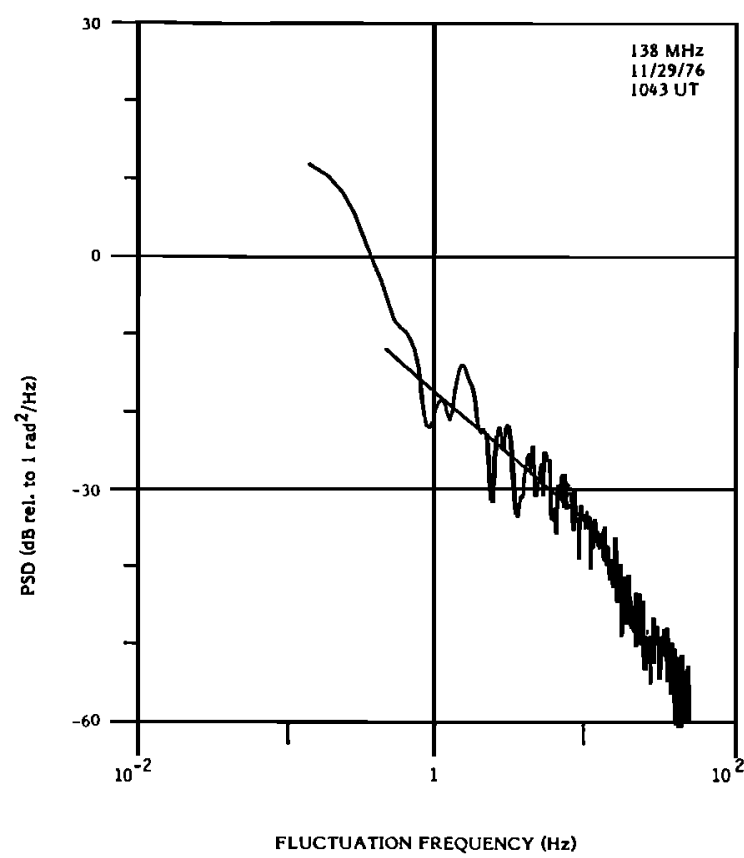

Fig. 5. VHF phase spectrum displaying a high-frequency downward break as well as the low-frequency upward (inverse) break noted in the two spectra of Figure 4.

sight near grazing incidence on the field [Briggs and Parkin, 1963]. Were a localized enhancement to increase $p$ artifically via nonstationarity, we should see such an increase near the magnetic zenith in Figure 6 , and we do not.

In contrast to the random pattern in Figure 6, the nighttime contour plot, shown in Figure 7, displays a decidedly ordered pattern. Surprisingly, however, it is ordered in off-meridian angle as well as in off-shell angle. That is, the $p$ increase observed in Figure 1 does not appear in Figure 7 as the expected horizontal band. Rather, the $p$ enhancement occurs preferentially away from the station's magnetic meridian.

The Wideband data base contains about twice as many nighttime data points as it does daytime points. In order to assess the statistical significance of differences observed between daytime and nighttime behaviors of $p$, the nighttime data population was decimated by using only every other chronologically ordered point in a given bin prior to plotting Figure 7. A total of 6012 spectra contributed to Figure 7, as compared with 6647 for Figure 6, and the numbers of points per bin were similar through the important central portions of the two plots. The full nighttime data population ( $p$ values from 11,811 spectra) was employed in Figure 8 in order to refine the presentation of $p$ behavior. Figure 8 reveals, even more clear- 
$\mathbf{p}$
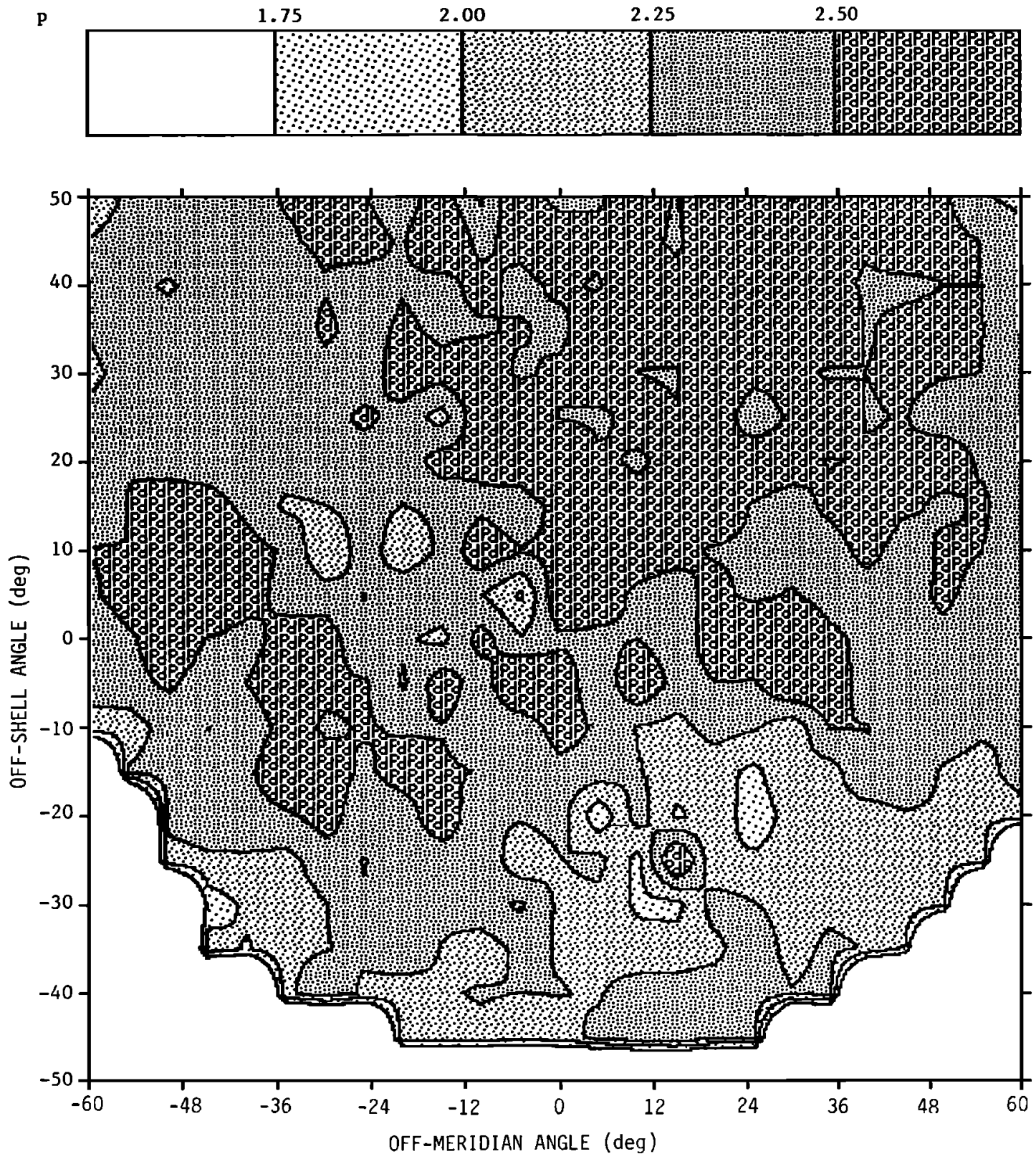

Fig. 6. Contour plot of VHF phase-spectral index, $p$, for all daytime Wideband data from Poker Flat. Gray scale appears at top. Coordinate system refers to angle, calculated at $350-\mathrm{km}$ altitude, between the line of sight and the local, $x$, magnetic meridian and $y$, magnetic $L$ shell.

ly than Figure 7, the tendency for the nighttime enhancement in $p$ to avoid the magnetic meridian.

Nighttime Wideband passes over Poker Flat progressed very nearly from geomagnetic north to south. The rectangular boxes on Figure 8 represent masks used for conveniently selecting pass segments for comparison of the different behaviors noted near and away from the magnetic meridian. In terms of rou- tine 20-s processing periods in the passes that we subsequently processed specially, we found 17 points from 6 passes in region 1,22 points from 5 passes in region 2 , and 28 points from 9 passes in region 3 . For detailed inspection, we selected periods of low $p$ from region 2 and periods of high $p$ from regions 1 and 3 . For instance, the pass illustrated in Figure 4 traversed region 3. Table 1 indicates, in terms of routine 
$\mathbf{p}$
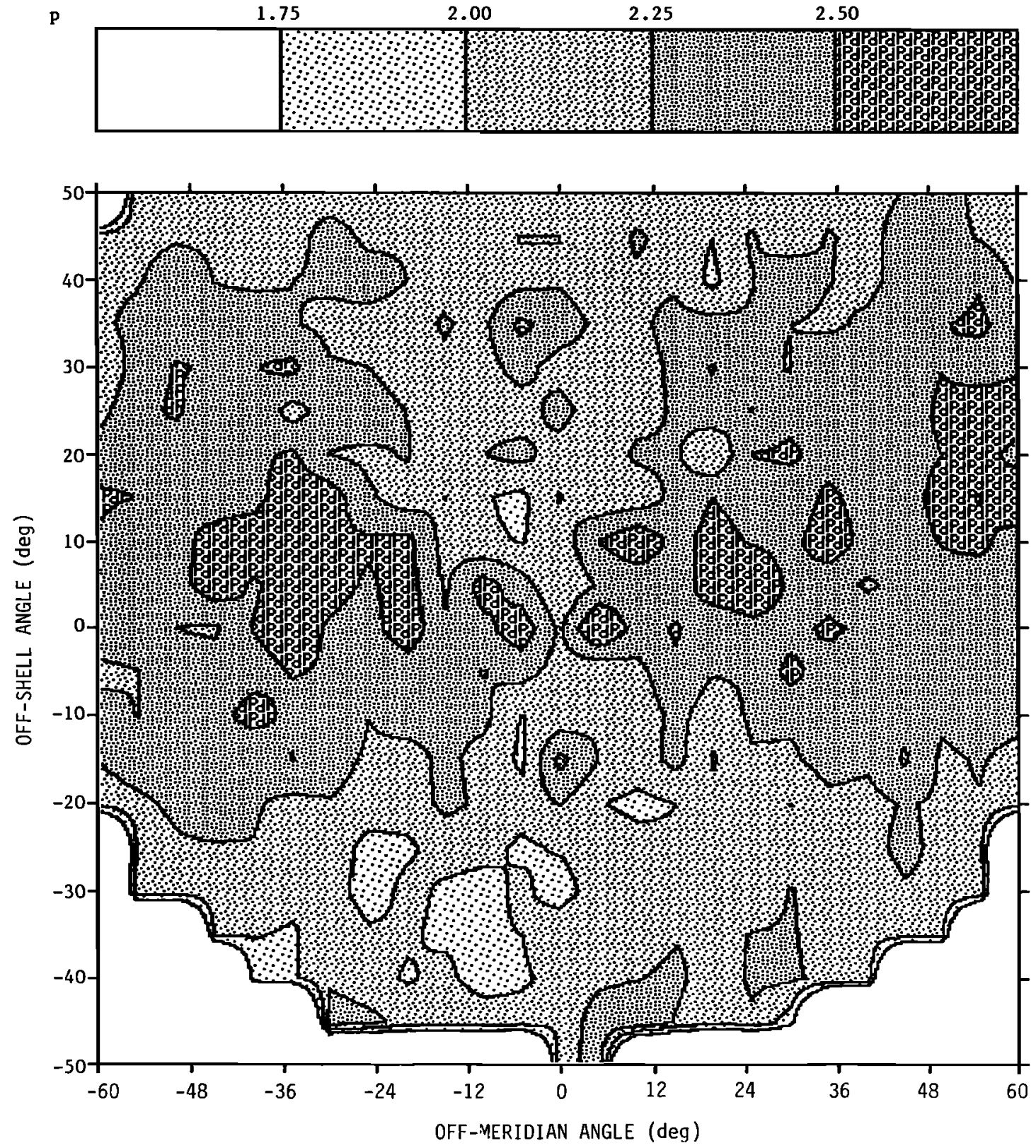

Fig. 7. Contour plot of $p$, in the same coordinate system as in Figure 6, for nighttime VHF data base decimated to provide statistical significance comparable to that of daytime-data population.

20-s periods, that the points obtained from the masked areas are consistent with the behavior of $p$ in the overall nighttime data population.

We selected 15 of the aforementioned 20 passes for detailed spectral analysis, including a preponderance showing the $p$ behavior illustrated in Figure 8, a few counter-examples, and one very weak scintillation pass as a check on noise effects. For each of the 15 passes, we plotted the phase time series for 5 or $6 \mathrm{~min}$ including the scintillation enhancement. The time series plots were used to hand select 20 -s periods showing reasonable statistical stationarity, for spectral analysis. Periods totally inside the region and totally outside the enhancement region were sought, and transition periods were identified.

Figure 9 shows some of the spectra computed after the foregoing selection. The cases were chosen as exemplary of behavior well off (Figure $9 a$, from 

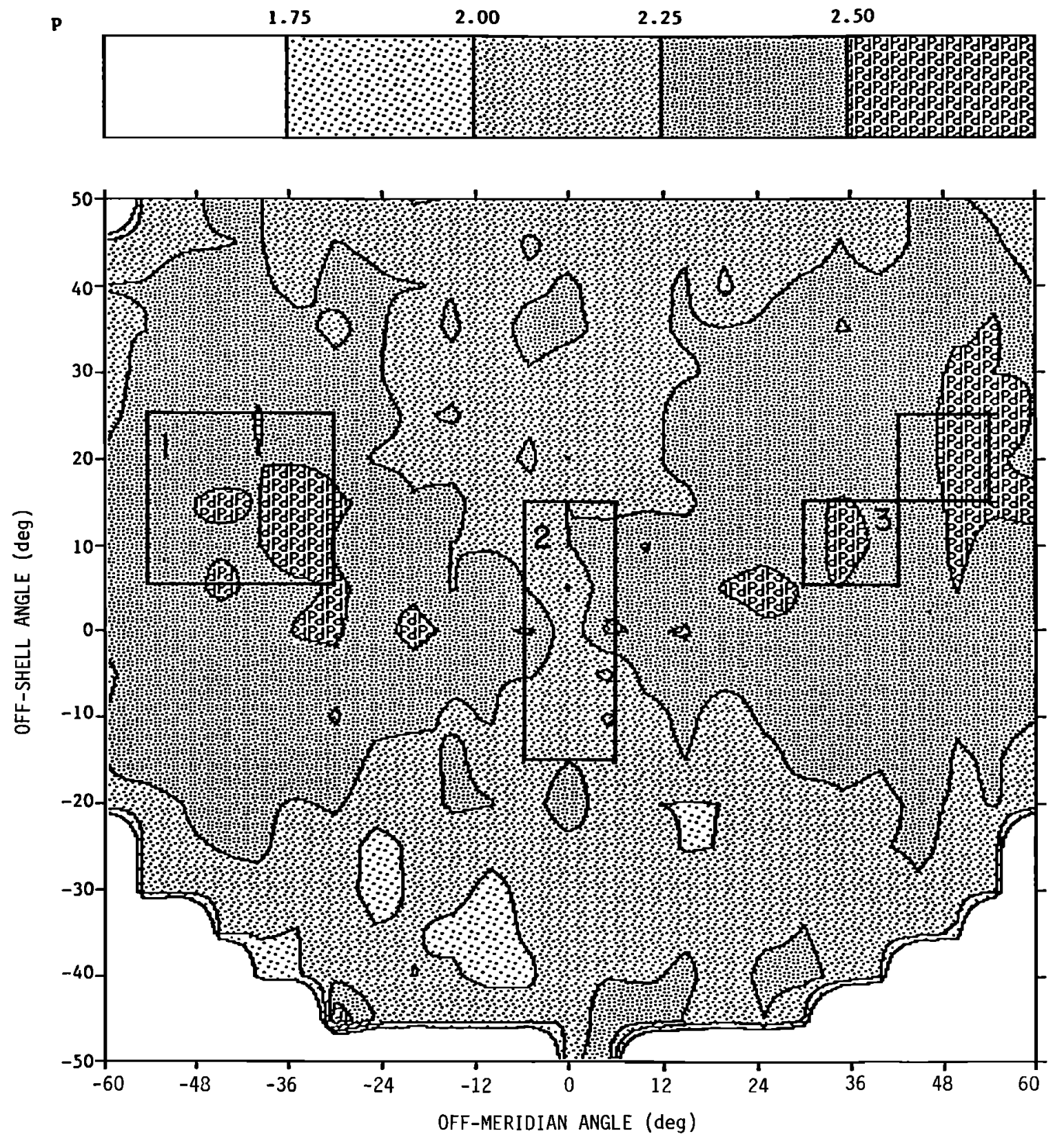

Fig. 8. Contour plot of $p$ using entire nighttime VHF Wideband data base from Poker Flat. Rectangles show regions of data selected for detailed spectral analysis.

region 3) and near (Figure $9 b$, from region 2) the magnetic meridian. The former (Figure 9a, pass 61-33) shows an ordered increase in $p$ through the off-meridian enhancement region. The latter (Figure $9 b$, pass 52-46) is an extreme example of nearmeridian behavior. Not only does $p$ remain at values well below the maximum achieved in the offmeridian case, but it actually decreases substantially in the strongest portion of the scintillation enhancement (as identified on the time series plot).

The spectra illustrated in Figure 9 were obtained by using a Blackman-Harris window. The first data point $(0.1 \mathrm{~Hz})$ was elevated by $3 \mathrm{~dB}$ to offset detrender suppression, and a five-bin centered smoother (modified to avoid end effects) has been applied. The straight lines superimposed on the spectra represent 
log linear best fits in the spectral band routinely employed for that purpose in Wideband summary processing. The spectra shown are quite well approximated by single-component power laws in that band and above, although several show some enhancement in a lower frequency band. The noise floor is at about $-70 \mathrm{~dB}$ on the scale indicated. The $p$ values derived from the fit procedure are indicated on the plots. The first and last spectra in both Figures $9 a$ and $9 b$ are from outside the geometrical scintillation enhancement. The central two spectra in each case are from the enhancements, and they show the opposite spectral-index behaviors described above. (Note that the central two spectra in Figure $9 b$ are from overlapped time periods in pass 52-46, which displayed a narrower enhancement than did pass 6133.) Qualitatively, it appears that the off-meridian enhancement preferentially affected large-scale structures, whereas all scales were enhanced (and especially small ones in the present example) near the magnetic zenith.

The peak enhancement in pass $52-46$ occurred as the line of sight passed within about $2^{\circ}$ of field alignment. For this special geometry and for irregularities highly elongated along the magnetic field, it is conceivable that the thick-medium limit usually employed in applying equation (1) might break down. In the opposite (thin-layer) limit, equation (1) becomes

$$
R_{\phi}(\zeta)=r_{e}^{2} \lambda^{2} L^{2} R_{\Delta N}(\zeta, 0)
$$

and the two-dimensional phase spectrum becomes identical with the two-dimensional irregularity spectrum. Instead of being averaged out, structure along the line of sight contributes the integral of all its spectral components. Indeed, in this limiting case, the line-of-sight integration described by (1) is fully coherent, and the phase perturbation goes as the square of the layer thickness.

Moreover, now when a strip scan is made of a phase pattern that may be characterized by a power law spectrum, the same one-dimensional spectral

TABLE 1. Distribution of Spectral Indices in Regions Identified in Figure 8

\begin{tabular}{cccc}
\hline & \multicolumn{3}{c}{ Number of Points } \\
\cline { 2 - 4 } Region & Total & $p>2.5$ & $p<2.0$ \\
\hline 1 & 17 & 7 & 3 \\
2 & 22 & 2 & 13 \\
3 & 28 & 8 & 2 \\
\hline
\end{tabular}

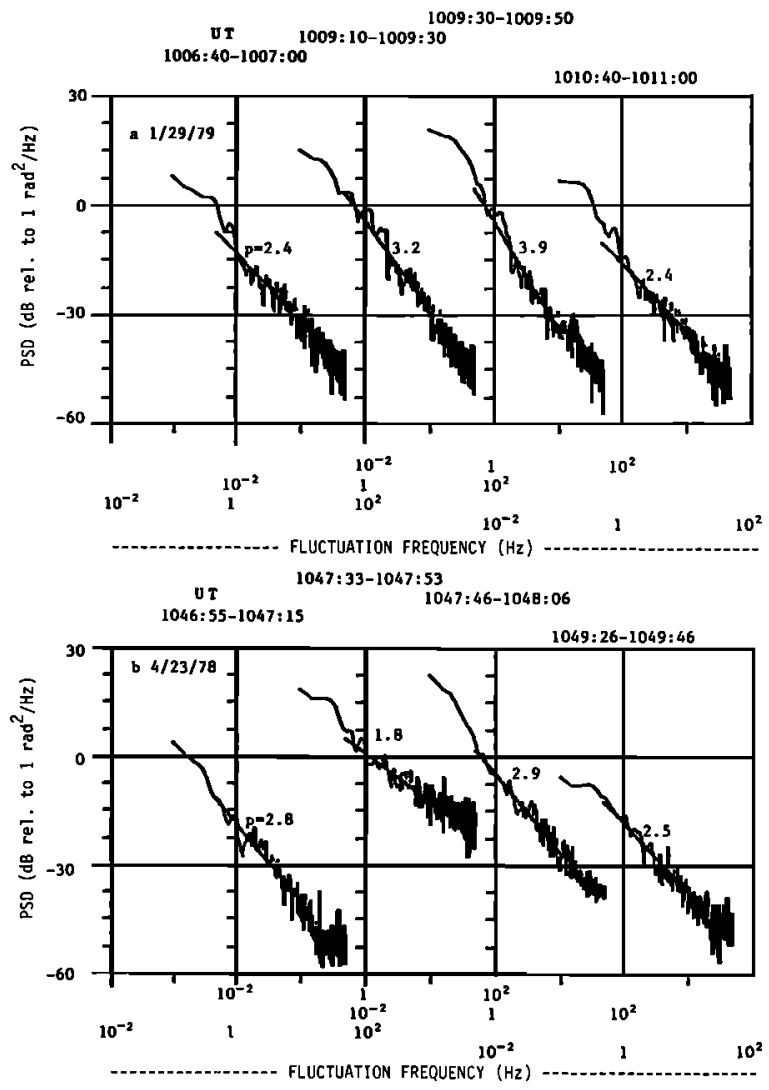

Fig. 9. VHF phase spectra from (a) an off-meridian and (b) a near-meridian Wideband pass over Poker Flat, both nighttime. Note opposite spectral behavior.

index, $p$, is obtained as would be reported by an in situ probe, namely $q$ instead of $q-1$. It may be that this relative subtlety of scintillation observations contributes a decrease in $p$ for passes very close to the magnetic zenith, such as that in Figure $9 b$. If it were a dominant effect, however, we should expect to find a region of decreased $p$ values centered on $(0,0)$ in Figure 6 (unless one is willing to postulate further that daytime irregularities are less elongated along the field than are nighttime ones), and we do not.

\section{CONCLUDING DISCUSSION}

As a result of the foregoing observations and analyses, we form the following two conclusions:

1. The spectra of phase scintillations in the nightside auroral oval, and most likely of the $F$ region irregularities that produce them, quite often show multiregime departures from single power law behavior. 
2. The nighttime subauroral scintillation enhancement, in addition to increasing the scintillation index, increases the phase spectral index for viewing angles east and west of the receiver's magnetic zenith, but not for nearly field aligned aspects.

A partial explanation for point 1 above (specifcally for the downward break in power-spectral density for wavelengths smaller than a few hundred meters) may be that offered by Basu et al. [1984] to explain their observation of increased in situ spectral indices "in regions of large auroral acceleration events." They suggested damping of small-scale irregularities by means of enhanced cross-field diffusion in highly conducting $E$ layer regions, as discussed by Vickrey and Kelley [1982]. More often than the downward break at small wavelengths, we observe an upward (inverse) break for wavelengths larger than a few kilometers.

To understand point 2 above, we offer a geometrical interpretation, since there is no reason to suppose anything geophysically unique about the viewer's magnetic zenith. One possibility is that the "sheetlike" irregularities [Rino et al., 1978] responsible for the scintillation enhancement [Fremouw et al., 1977] are confined primarily to the large-scale end of the scintillation-producing spectrum. That is, we suggest that the smaller-scale irregularities are more nearly axially symmetric than are the large ones. An idealized picture would be of small-scale "rods" imbedded in large-scale "sheets."

The scintillation enhancement has been defined mostly in terms of the integral, $\sigma_{\phi}^{2}$, of the phase spectrum. This phase variance is thought to be enhanced by quasi-coherent addition of phase perturbations as a radio wave propagates along an elongated axis of (sheetlike or rodlike) irregularities [Singleton, 1970a, b]. The same process would occur for the rod/sheet mix postulated in the foregoing paragraph. For such a mix, however, the phase spectrum would be uniformly enhanced only near the magnetic zenith, while there would be preferential enhancement of the largescale end of the spectrum at large off-meridian angles (and small off-shell angles). For the later geometry, quasi-coherent phase addition would arise in the (large-scale) sheetlike irregularities but not in the (small-scale) rodlike irregularities.

A likely sequence of events that could lead to the postulated scale-size-dependent anisotropy would be one initiated by structured, soft electron precipitation [Tanskanen et al., 1981] setting up latitudinally nonuniform $F$ layer ionization, such as that observed by Muldrew and Vickrey [1982] by means of incoherent scatter, and by Rino and Owen [1980] and Leitinger et al. [1982] as TEC enhancements. This $L$ shellaligned large-scale structure would form a "reservoir" spectral regime from which smaller-scale structure would bifurcate into a cascade regime by means of convective instability.

As a new element in the picture, the $p$ behavior reported here may indicate a trend toward cross-field isotropy in the cascade regime. Thus, there would be no need for nonlinear production of $L$ shell-aligned structures by convective processes, which do not seem to be a dominant result of computer simulations [Keskinen and Ossakow, 1982, and references therein]. We envision a multistage cascade process in which each generation produces structure not only smaller than itself but also oriented perpendicular to its own dominant alignment (parallel to its own dominant gradient). Many generations taken together would display cross-field isotropy, but the largest (reservoir regime) structures would remain anisotropic.

For propagation calculations, the foregoing picture could be modeled by means of a two-component spectrum. Signal-statistically, the situation might be likened to the two-component model described by Fremouw et al. [1980], in which the large-scale component is responsible for large phase perturbations and geometrical-optics focusing and defocusing, while the small-scale component produces diffractive scatter. Now, however, the two components would have a phenomenological basis also, distinguishable not only in scale size but in degree of anisotropy.

Because Wideband was in a sun-synchronous orbit passing over Poker Flat in the midnight sector, we cannot unequivocally conclude that the behavior illustrated in Figure 8 is of geometric origin. That is, the geometric grid used there is not dissimilar from a magnetic latitude-time grid centered approximately on $65^{\circ}$ and 2300 (premidnight to the right, postmidnight to the left). Thus, one could suppose $p$ to be ordered with the high-latitude convection pattern, especially if credence is given to the tendency for the large- $p$ regions to form an ovallike pattern extending farthest south in the midnight sector. Moreover, even if our geometrical interpretation is correct, we emphasize that our observations are limited to the approximately midnight sector of the auroral oval. The spectral behavior in other sectors could be different.

If the latter, strictly geophysical, interpretation is correct, then in situ data should show a pattern of $p$ ordered in magnetic latitude and time; if our geometrical interpretation is correct, there should be no 
such ordering. These two hypotheses also should be testable with scintillation data from satellites that precess in magnetic time. Such precession was an important consideration in selecting an orbit for the DNA HiLat satellite [Fremouw et al., 1983], and its capability to provide simultaneous scintillation, in situ, and particle precipitation observations should facilitate such a test.

Acknowledgments. We thank C. Rino and R. Livingston of SRI International for cooperation in continuing to make data from the DNA Wideband Satellite fully available to us. The work reported here was supported by the Defense Nuclear Agency under contract DNA001-81-C-0092.

\section{REFERENCES}

Basu, S., S. Basu, J. P. McClure, W. B. Hanson, and H. E. Whitney, High-resolution topside in situ data of electron densities and $\mathrm{VHF} / \mathrm{GHz}$ scintillations in the equatorial region, J. Geophys. Res., 88, 403, 1983.

Basu, S., S. Basu, and E. MacKenzie, $F$ region electron density irregularity spectra near auroral acceleration and shear regions, J. Geophys. Res., 89(A7), 5554-5564, 1984.

Briggs, B. H., and I. A. Parkin, On the variation of radio star and satellite scintillations with zenith angle, J. Atmos. Terr. Phys., 25(6), 339-366, 1963.

Cronyn, W. M., The analysis of radio scattering and space-probe observations of small-scale structure in the interplanetary medium, Astrophys. J., 161, 755-762, 1970.

Fremouw, E. J., and J. M. Lansinger, Dominant configurations of scintillation-producing irregularities in the auroral zone, J. Geophys. Res., 86(A12), 10,087-10,093, 1981.

Fremouw, E. J., C. L. Rino, R. C. Livingston, and M. C. Cousins, A persistent subauroral scintillation enhancement observed in Alaska, Geophys. Res. Lett., 4(11), 539, 1977.

Fremouw, E. J., R. L. Leadabrand, R. C. Livingston, M. D. Cousins, C. L. Rino, B. C. Fair, and R. A. Long, Early results from the DNA Wideband satellite experiment: Complex-signal scintillation, Radio Sci., 13(1), 167-187, 1978.

Fremouw, E. J., R. C. Livingston, and D. A. Miller, On the statis- tics of scintillating signals, J. Atmos. Terr. Phys., 42, 717-731, 1980.

Fremouw, E. J., et al., The HiLat program Eos Trans. $A G U$, 64(18), 163-170, 1983.

Harris, F. J., On the use of windows for harmonic analysis with the discrete Fourier transform, Proc. IEEE, 66, 55-83, 1978.

Keskinen, M. J., and S. L. Ossakow, Nonlinear evolution of convecting plasma enhancements in the auroral ionosphere, II, Small scale irregularities, NRL Memo. Rep. 4323, Nav. Res. Lab., Washington, D. C., 1982.

Leitinger, R., G. K. Hartmann, W. Degenhardt, A. Hedberg, and $P$. Tanskanen, The electron content of the ionosphere and the southern boundary of diffuse aurora, J. Atmos. Terr. Phys., 44(4), 369, 1982.

Muldrew, D. B., and J. F. Vickrey, High-latitude $F$ region irregularities observed simultaneously with ISIS 1 and the Chatanika radar, J. Geophys. Res., 87(A10), 8263, 1982.

Rino, C. L., and E. J. Fremouw, The angle dependence of singly scattered wavefields, J. Atmos. Terr. Phys., 39, 859, 1977.

Rino, C. L., and J. Owen, The structure of localized nighttime auroral zone scintillation enhancements, J. Geophys. Res., 85(A6), 2941-2948, 1980.

Rino, C. L., R. C. Livingston, and S. J. Matthews, Evidence for sheetlike auroral ionospheric irregularities, Geophys. Res. Lett., 5(12), 1039, 1978.

Rino, C. L., R. T. Tsunoda, J. Petriceks, R. C. Livingston, M. C. Kelley, and K. D. Baker, Simultaneous rocket-borne beacon and in situ measurements of equatorial spread $F$ : Intermediate wavelength results, J. Geophys. Res., 86(A4), 2411-2420, 1981.

Singleton, D. G., The effect of irregularity shape on radio star and satellite scintillations, J. Atmos. Terr. Phys., 32, 315-343, 1970 a.

Singleton, D. G., Dependence of satellite scintillations on zenith angle and azimuth, J. Atmos. Terr. Phys., 32, 789-803, 1970 b.

Tanskanen, P. J., D. A. Hardy, and W. J. Burke, Spectral characteristics of precipitating electrons associated with visible aurora in the premidnight oval during periods of substorm activity, $J$. Geophys. Res., 86(A3), 1379-1395, 1981.

Vickrey, J. F., and M. C. Kelley, The effects of a conducting $E$ layer on classical $F$ region cross-field plasma diffusion, $J$. Geophys. Res., 87, 4461, 1982.

E. J. Fremouw, J. M. Lansinger, and J. A. Secan, Physical Dynamics, Inc., 300 120th Ave., N.E., Suite 220, Building 7, Bellevue, WA 98005. 